\title{
Secure Quantum Secret Sharing Based on Reusable GHZ States as Secure Carriers
}

\author{
Jie SONG and Shou ZHANG* \\ Department of Physics, College of Science, \\ Yanbian University, Yanji, Jilin 133002, PR China
}

\begin{abstract}
We show a potential eavesdropper can eavesdrop whole secret information when the legitimate users use secure carrier to encode and decode classical information repeatedly in the protocol [proposed in Bagherinezhad S and Karimipour V 2003 Phys. Rev. A 67 044302]. Then we present a revised quantum secret sharing protocol by using Greenberger-Horne-Zeilinger state as secure carrier. Our protocol can resist Eve's attack.

PACS numbers: 03.67. Hk, 03.65. Ud
\end{abstract}

Keywords: GHZ state, controlled-Not operation, Hadamard operation

Quantum secret sharing (QSS) is a generalization of classical secret sharing to a quantum scenario. The basic idea of QSS is: After Alice distributes her secret message to the receivers (Bob, Charlie, ...), all the receivers can obtain the secret message simultaneously by collaboration. Since Hillery et al. 1] proposed the pioneering QSS work, QSS has attracted a widespread attention and progressed quickly [2, 3, 4, 5, 6, 7, 8, 9] over the past several years. In 2001, Guo et al. [10] proposed a quantum key distribution protocol by using quantum key to encode and decode classical information. The quantum key can be used repeatedly. Based on Guo's protocol, Karimipour et al. 11] proposed a QSS protocol by using Greenberger-Horne-Zeilinger (GHZ) states as secure carriers.

Recently, Gao et al. [12] present an eavesdropping protocol by which Eve can obtain odd numbered data bits of Karimipour's protocol [1]. Karimipour [13] shows that the quantum bits in odd rounds can be discarded by legitimate users. Only if Eve can get the whole information, this protocol can be proved insecure. In this paper, we propose another eavesdropping method to the protocol in Ref. 11]. Eve can get the whole information

\footnotetext{
*E-mail: szhang@ybu.edu.cn
} 
without being detected by the legitimate users. Then we present a revised QSS protocol by using GHZ state to encode and decode classical information. Our QSS protocol can be robust against Eve's attack.

First of all, let us give a brief review of Karimipour's protocol [11]. In the beginning, Alice, Bob and Charlie have shared a GHZ state $|\psi\rangle=\frac{1}{\sqrt{2}}(|0,0,0\rangle+|1,1,1\rangle)_{a, b, c}$ as carrier (used subscripts a, b and c on the states and operators for Alice, Bob and Charlie, respectively), and the data bits Alice wants to distributed to Bob and Charlie can be represented by $q_{1}, q_{3}, q_{5}, \cdots$ (where $q=0$ or 1 ) in odd round and $q_{2}, q_{4}, q_{6}, \cdots$ (where $q \rightarrow \bar{q}=|q\rangle_{12}=$ $\frac{1}{\sqrt{2}}\{|0, q\rangle+|1, q \oplus 1\rangle\}$ and " $\oplus$ " is performed modulo 2$)$ in even round. In odd round, Alice entangles the state $|q, q\rangle_{1,2}$ to the carrier by performing controlled-NOT (CNOT) operations $C_{a 1}, C_{a 2}$ and send them to Bob and Charlie $\left(C_{a 1}\right.$ is the controlled-NOT gate and is specified by two subscripts, the first one is the control bit, the second one is the target bit). Bob and Charlie act on this state by the operations $C_{b 1}, C_{c 2}$ to read independently his own bit. In even round, Alice performs $C_{a 1}$ to entangle the state $|\bar{q}\rangle_{12}$ to the carrier. Bob and Charlie disentangle the state $|\bar{q}\rangle_{12}$ from the carrier by the operations $C_{b 1}, C_{c 2}$. Bob and Charlie can gain Alice's information by collaboration. Now let us describe our eavesdropping strategy to eavesdrop the whole information of [1]]. Eve prepares two qubits $\left(e_{1}\right.$ and $\left.e_{2}\right)$ in state $|0\rangle$ as her two ancilla.

(1) In the first round, according to the method in Ref. [12], Eve intercepts the first sending qubit which is denoted by subscript 1 , and then performs a controlled-NOT (CNOT) operation $C_{1 e_{1}}$ on this qubit and her ancilla after Alice sent the particles. This state of these qubits can be specified by

$$
\left|\psi^{1}\right\rangle= \begin{cases}\frac{1}{\sqrt{2}}(|0,0,0,0,0,0\rangle+|1,1,1,1,1,1\rangle)_{a, b, c, e_{1}, 1,2} & \left(q_{1}=0\right), \\ \frac{1}{\sqrt{2}}(|0,0,0,1,1,1\rangle+|1,1,1,0,0,0\rangle)_{a, b, c, e_{1}, 1,2} & \left(q_{1}=1\right),\end{cases}
$$

and then Eve resends it to Bob. Here superscript 1 denotes the first round. The value of $q_{n}$ denotes Alice's sending information. Bob and Charlie can get the right information when they disentangle the sending qubit from the carrier.

(2) In the second round, as Alice, Bob and Charlie perform a Hadamard gate on their particles, respectively, Eve also performs the same operation on his ancilla $e_{1}$. After Alice entangles the entangled state $\overline{q_{2}}$ to the carrier, she sends the two particles (1 and 2) to Bob and Charlie, then Eve intercepts the two sent qubits and performs two CNOT operations 
$C_{1 e_{2}}, C_{2 e_{2}}$. The qubit $e_{2}$ is also entangled to the carrier. Eve performs a CNOT operation $C_{e_{1} 1}$. She resends them to Bob and Charlie. After Bob and Charlie performed two CNOT operations $C_{b 1}, C_{c 2}$ to disentangle their receiving particles from the carrier and measured the qubits 1 and 2, they cooperate to get the value of $q_{2}$ and can't detect the presence of Eve, while leaving the state

$$
\left|\psi^{2}\right\rangle \rightarrow\left\{\begin{array}{cc}
\frac{1}{2 \sqrt{2}}(|0,0,0,0,0\rangle+|0,0,1,1,0\rangle+|0,1,0,1,0\rangle+|0,1,1,0,0\rangle+|1,0,0,1,1\rangle \\
+|1,0,1,0,1\rangle+|1,1,0,0,1\rangle+|1,1,1,1,1\rangle)_{a, b, c, e_{1}, e_{2}} \quad\left(q_{2}=0, q_{1}=0\right), \\
\frac{1}{2 \sqrt{2}}(|0,0,0,0,1\rangle+|0,0,1,1,1\rangle+|0,1,0,1,1\rangle+|0,1,1,0,1\rangle+|1,0,0,1,0\rangle \\
+|1,0,1,0,0\rangle+|1,1,0,0,0\rangle+|1,1,1,1,0\rangle)_{a, b, c, e_{1}, e_{2}} \quad\left(q_{2}=1, q_{1}=0\right), \\
\frac{1}{2 \sqrt{2}}(|0,0,0,0,0\rangle-|0,0,1,1,0\rangle-|0,1,0,1,0\rangle+|0,1,1,0,0\rangle-|1,0,0,1,1\rangle \\
+|1,0,1,0,1\rangle+|1,1,0,0,1\rangle-|1,1,1,1,1\rangle)_{a, b, c, e_{1}, e_{2}} \quad\left(q_{2}=0, q_{1}=1\right), \\
\frac{1}{2 \sqrt{2}}(|0,0,0,0,1\rangle-|0,0,1,1,1\rangle-|0,1,0,1,1\rangle+|0,1,1,0,1\rangle-|1,0,0,1,0\rangle \\
+|1,0,1,0,0\rangle+|1,1,0,0,0\rangle-|1,1,1,1,0\rangle)_{a, b, c, e_{1}, e_{2}} \quad\left(q_{2}=1, q_{1}=1\right) .
\end{array}\right.
$$

(3) In the third round, Eve can eavesdrop the odd round's key bit. Firstly, Eve also performs a Hadamard gate on each of her two ancilla, when Alice, Bob and Charlie perform a Hadamard gate on their respective particles. The state of Alice, Bob, Charlie and Eve can be expressed as

$$
\left|\psi^{3}\right\rangle \rightarrow\left\{\begin{array}{lc}
\frac{1}{2}(|0,0,0,0,0\rangle+|1,1,1,1,0\rangle+|1,0,0,0,1\rangle+|0,1,1,1,1\rangle)_{a, b, c, e_{1}, e_{2}} \quad\left(q_{1}=0, q_{2}=0\right), \\
\frac{1}{2}(|0,0,0,0,0\rangle+|1,1,1,1,0\rangle-|1,0,0,0,1\rangle-|0,1,1,1,1\rangle)_{a, b, c, e_{1}, e_{2}} \quad\left(q_{1}=0, q_{2}=1\right), \\
\frac{1}{2}(|1,1,1,0,0\rangle+|0,0,0,1,0\rangle+|0,1,1,0,1\rangle+|1,0,0,1,1\rangle)_{a, b, c, e_{1}, e_{2}} \quad\left(q_{1}=1, q_{2}=0\right), \\
\frac{1}{2}(|1,1,1,0,0\rangle+|0,0,0,1,0\rangle-|0,1,1,0,1\rangle-|1,0,0,1,1\rangle)_{a, b, c, e_{1}, e_{2}} \quad\left(q_{1}=1, q_{2}=1\right),
\end{array}\right.
$$


After Alice sent out the two qubits, Eve intercepts the two qubits and performs four CNOT operations $C_{e_{1} 1}, C_{e_{2} 1}$ and $C_{e_{1} 2}, C_{e_{2} 2}$. Eve makes a measurement on one of two qubits (1 and 2) and gets the value of $q_{3} \oplus q_{1}$. After Eve performs $C_{e_{1} 1}$ and $C_{e_{1} 2}$, she resends the two qubits (1 and 2) to Bob and Charlie. Bob and Charlie perform $C_{b 1}$ and $C_{c 2}$ on their respective qubits. We conclude that Bob and Charlie get correct result.

(4) In the fourth round, Alice, Bob, Charlie and Eve perform a Hadamard gate operation on their respective qubits. As a result, the state of whole system will be converted into Eq. (2). In this round, Eve can eavesdrop the key bit of even round without being detected. Suppose information bit of Alice is $q_{4}=|\bar{k}\rangle_{12}(k=0$ or 1$)$, after Alice performed a CNOT operation $C_{a 1}$ and sent the two qubits to Bob and Charlie, Eve intercepts the two qubits and performs a CNOT operation $C_{e_{2} 1}$. The two qubits have disentangled from the carrier. Eve can perform two single particle measurements on the two qubits (1 and 2) and get the value of $q_{2} \oplus q_{4}$. Afterward, she prepares two qubits in the state $\left|\overline{q_{2} \oplus q_{4}}\right\rangle_{1^{\prime}, 2^{\prime}}$ and performs CNOT operations $C_{e_{2} 1^{\prime}}, C_{e_{1} 1^{\prime}}$ and sends them to Bob and Charlie, respectively. Bob and Charlie act on their respective qubits by the operations $C_{b 1^{\prime}}$ and $C_{c 2^{\prime}}$, so they can get the correct information.

(5) In next odd and even rounds, Eve uses the same strategy in step (3) and (4). So Eve can get the information ( $q_{1} \oplus q_{3}, q_{1} \oplus q_{5}, \cdots$ and $\left.q_{2} \oplus q_{4}, q_{2} \oplus q_{6}, \cdots\right)$. As long as any odd and even numbered data bits are announced by Alice, Eve could obtain Alice's full information.

Comparing with Gao's strategy [12] our strategy improves the efficiency of eavesdropping. We can prove that the protocol [1] is insecure by our eavesdropping strategy. So we modify this protocol [11] as follows:

(1) Alice, Bob and Charlie have shared one three-particle state $|\chi\rangle$ as carrier (the state can be expressed as $\left.|\chi\rangle=\frac{1}{\sqrt{2}}(|0,0,0\rangle+|1,1,1\rangle)_{a, b, c}\right)$. The following property will be used:

$$
H \otimes H \otimes H|\chi\rangle=|G\rangle=\frac{1}{2}(|0,0,0\rangle+|1,1,0\rangle+|1,0,1\rangle+|0,1,1\rangle)_{a, b, c} .
$$

(2) Before Alice sends her information qubits to Bob and Charlie, she decides if she performs a Hadamard gate on her qubit a. If Alice performs a Hadamard gate on her qubit, she chooses two qubits which are in two-qubit state $q \rightarrow \bar{q}=|q\rangle_{12}=\frac{1}{\sqrt{2}}(|0, q\rangle+|1, q \oplus 1\rangle)$ for sending a classical bit. Alice performs one single CNOT gate $C_{a 1}$ to entangle this state to the carrier

$$
|\psi\rangle=\frac{1}{2}\{(|0, \bar{q}\rangle+|1, \overline{q \oplus 1}\rangle) \otimes|0,0\rangle+(|0, \bar{q}\rangle-|1, \overline{q \oplus 1}\rangle) \otimes|1,1\rangle\}_{a, 1,2, b, c}
$$


and sends the two qubits to Bob and Charlie. After Bob and Charlie have received the two qubits, Alice informs that she did a Hadamard gate. Then Bob and Charlie also perform a hadamard gate accordingly. This state of these qubits will be specified by

$$
|\psi\rangle=\frac{1}{2}\{|0, \bar{q}\rangle \otimes(|0,0\rangle+|1,1\rangle)+|1, \overline{q+1}\rangle \otimes(|0,1\rangle+|1,0\rangle)\}_{a, 1,2, b, c} .
$$

Bob and Charlie perform two CNOT operations $C_{b 1}$ and $C_{c 2}$ on their respective qubits to disentangle qubits 1 and 2 .

(2') If Alice does't perform a Hardamard gate on his qubit a, she will choose two single particles which are in the state $\left|q_{1}\right\rangle$ and $\left|q_{2}\right\rangle\left(q_{1}, q_{2} \in(0,1)\right)$. The classical bit is the value of $q_{1} \oplus q_{2}$. Alice performs one CNOT gate $C_{a 1}$ or $C_{a 2}$ to entangle one of the two particles to the carrier. Suppose Alice performs $C_{a 1}$. The state of these qubits can be expressed as

$$
|\psi\rangle=\frac{1}{\sqrt{2}}\left\{\left(\left|0,0,0, q_{1}\right\rangle+\left|1,1,1, q_{1} \oplus 1\right\rangle\right) \otimes\left|q_{2}\right\rangle\right\}_{a, b, c, 1,2} .
$$

Alice sends the two qubits to Bob and Charlie. After Bob and Charlie have received the two qubits, Alice informs that she did not perform a Hadamard gate and performed a CNOT gate $C_{a 1}$. Then Bob perform $C_{b 1}$ to disentangle his receiving qubit 1 .

(3) Bob and Charlie performs single particle measurement on their respective particles (1 and 2). Only one of them publicly announces the measurement result, and the other will add the publicly announced result to his own measurement result to get Alice's information.

(4) In next round, Alice know the three-particle state is in $|\chi\rangle$ or $|G\rangle$ according to the number of Hadamard gate she has performed. Alice, Bob and Charlie do similar operations from step (2) to (4).

(5) At last, Alice publicly announces part of classical bits through a classical channel. They compare the results and analyze the error rate. If the error rate is reasonably low, they can believe the security of the process. Otherwise they terminate the QSS and start next one form step (1).

Now, let us discuss the security of the revised protocol. For convenience, we call Gao's attack strategy in [12] A1, and call our attacking strategy A2. Suppose Eve intends to use A1 to attack. Eve intercepts one of the two qubits 1 after Alice sent the qubits. Eve prepares a qubit in the state $|0\rangle$ as her ancilla e. She performs a CNOT operation $C_{1 e}$. Eve doesn't know that the qubits sent by Alice were in single particle state or two-qubit state. If the qubits sent by Alice are in two-qubit state (i.e., $|\overline{0}\rangle$ ), the state of whole system will 
be converted into

$$
|\psi\rangle=\frac{1}{2}\{|0\rangle \otimes(|0,0,0\rangle+|1,1,1\rangle) \otimes|0,0\rangle+|1\rangle \otimes(|1,0,1\rangle+|0,1,0\rangle) \otimes|1,1\rangle\}_{a, 1,2, e, b, c, \cdot}
$$

After Bob and Charlie received Alice's sending qubits, Alice announces that she performed a Hadamard gate. Bob and Charlie also perform a Hadamard gate on the qubits b and c. Then they perform a CNOT operation $C_{b 1}$ and $C_{c 2}$ and make a single particle measurement on their qubits (1 and 2), respectively. The state of these qubits will be converted into one of the two states:

$$
|\psi\rangle=\left\{\begin{array}{l}
\frac{1}{2}(|0,0,0,0\rangle+|0,1,1,1\rangle+|1,0,1,0\rangle+|1,1,0,1\rangle)_{a, b, c, e} \\
\frac{1}{2}(|0,0,0,1\rangle+|0,1,1,0\rangle+|1,0,1,1\rangle+|1,1,0,0\rangle)_{a, b, c, e}
\end{array}\right.
$$

In next round, when Alice sends single particles as information qubits to Bob and Charlie, the eavesdropping behavior makes error in comparing the data bits jointly received by Bob and Charlie with those sent by Alice. If Alice sends single particles as information qubits in the beginning, Eve performs a CNOT operation $C_{1 e}$ on the intercepted qubit and her ancilla. Eve might entangle his ancilla to the carrier. But in next round Alice doesn't announce if she has performed a hadamard gate until Bob and Charlie received her sending qubits. Owing to Eve doesn't know how to operate the intercepted qubit, she inevitably introduces error. So Eve can't get any useful information and her eavesdropping behavior will be detected by the legitimate users.

Suppose Eve wants to entangle his ancilla to the carrier by using the method of even round in A2 to attack. After Alice sent his two information bits to Bob and Charlie, Eve intercepts the two sending qubits and performs two CNOT operations $C_{1 e}, C_{2 e}$ on the two particles and her ancilla. Eve's aim is to entangle the state of his ancilla to the state $|G\rangle$. When Alice sends single particles as information qubits and performs a CNOT gate $C_{a 1}$, the state of whole system can be expressed as

$$
|\phi\rangle=\frac{1}{\sqrt{2}}\left\{\left(\left|0,0,0, q_{1}, q_{1} \oplus q_{2}\right\rangle+\left|1,1,1, q_{1} \oplus 1, q_{1} \oplus q_{2} \oplus 1\right\rangle\right) \otimes\left|q_{2}\right\rangle\right\}_{a, b, c, 1, e, 2} .
$$

When the two qubits sent by Alice are in two-qubit state, Eve can also entangle her ancilla to the carrier. Then the state of these qubits can be as follows

$\left|\phi^{\prime}\right\rangle=\frac{1}{2}\left\{\left(|0,0,0, \bar{q}, q\rangle+\left(|1,1,0, \overline{q \oplus 1}, q \oplus 1\rangle+(|1,0,1, \overline{q \oplus 1}, q \oplus 1\rangle+(|0,1,1, \bar{q}, q\rangle)\}_{a, b, c, 1,2, e}\right.\right.\right.$. 
When Alice sends his information qubits in next round, Eve can not know these information qubits of Alice are in single particle state or two qubit-state ahead of time. So Eve should not decide how to operate and eavesdrop information.

Even if one of the legitimate users (Bob) is dishonest and wants to eavesdrop Alice's information by intercept-and-resend attack strategy, he can't also eavesdrop the information without being detected. For example, after Alice sent two information qubits, Bob intercepts the two qubits and sends his qubit 2' to charlie. Charlie would take the qubit 2' for Alice's qubit 2. If Alice sends two single particles as information qubits and entangles Bob's qubit 1 to the carrier, she announces her operations after confirming Bob and Charlie have received their qubits. Then Bob can perform a CNOT gate $C_{b 1}$ and two single particle measurements on the qubits 1 and 2. So he can get the value of $q_{1} \oplus q_{2}$. Bob also knows the value of $q_{2^{\prime}}$, and then he can announce the value of $q_{1^{\prime}}$ according to $q_{1} \oplus q_{2}=q_{1^{\prime}} \oplus q_{2^{\prime}}$. As a result Bob can get the secret information without being detected. But when Alice entangles Charlie's qubit 2 to the carrier, Charlie should perform a CNOT gate $C_{c 2^{\prime}}$ and make a measurement on her receiving qubit 2'. She will get the value of $q_{2^{\prime}}$ or $q_{2^{\prime}} \oplus 1$. Bob can still gain the value of $q_{1} \oplus q_{2}$ after knowing Alice's operations. But he can't know the measurement result of Charlie is $q_{2^{\prime}}$ or $q_{2^{\prime}} \oplus 1$. This would lead to $50 \%$ difference between the bit recovered by Charlie and the information bit Alice has sent. If Bob intercepts the two qubits which are in two-qubit state, he can't get the right information according to Eq (44). His eavesdropping behavior will be detected by the legitimate users.

In summary, firstly, in our revised QSS protocol the secure carrier can be used repeatedly to send secret information when there is no presence of Eve. Secondly, Eve can't eavesdrop the secret information by entangling her ancilla to the carrier. The dishonest one of the two legitimate users can't gain information without being detected. The revised protocol is unconditionally secure. Thirdly, in the original protocol [11] Alice encodes information bits as a state $|q, q\rangle$ for sending odd bit. Bob and Charlie can recover the information bit without each other's assistance. In our protocol, Bob and Charlie must cooperate to gain Alice's information in every round. So our protocol improves the efficiency of the secret sharing. Furthermore, when Alice sends $N$ bits information to Bob and Charlie, they will perform $N$ Hadamard gates in the original protocol. In our protocol they will perform Hadamard gate 
operation randomly. Our protocol decreases the number of Hadamard gate operations.

[1] Hillery M, Buzek V and Berthiaume A 1999 Phys. Rev. A 591829

[2] Tittel W, Zbinden H and Gisin N 2001 Phys. Rev. A 63042301

[3] Gottesman D 2000 Phys. Rev. A 61042311

[4] Nascimento A C A, Quade J M and Imai H 2001 Phys. Rev. A 64042311

[5] Guo G P and Guo G C 2003 Phys. Lett. A 310247

[6] Deng F G and Long G L 2004 Phys. Rev. A 69052319

[7] Zhang Z J and Man Z X 2005 Chin. Phys. Lett. 221588

[8] Deng F G, Zhou H Y and Long G L 2005 Phys. Lett. A 337329

[9] Cheng Z H and Liu X M 2005 Chin. Phys. 141821

[10] Zhang Y S, Li C F and Guo G C 2001 Phys. Rev. A 64024302

[11] Bagherinezhad S and Karimipour V 2003 Phys. Rev. A 67044302

[12] Gao F, Guo F Z, Wen Q Y, and Zhu F C 2005 Phys. Rev. A 72036302

[13] Karimipour V 2005 Phys. Rev. A 72056301 